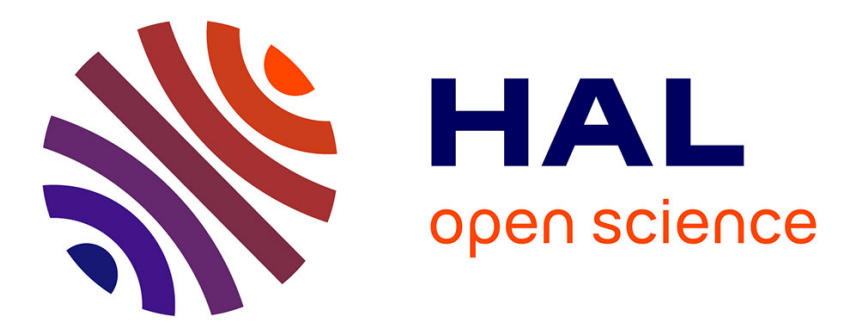

\title{
Polarisation-dependent extraction properties of bare fiber probes
}

\author{
T. Grosjean, Mathieu Mivelle Mivelle, G.W. Burr
}

\section{To cite this version:}

T. Grosjean, Mathieu Mivelle Mivelle, G.W. Burr. Polarisation-dependent extraction properties of bare fiber probes. Optics Letters, 2010, 35 (3), pp.357-359. 10.1364/OL.35.000357 . hal-00463216

\section{HAL Id: hal-00463216 https://hal.science/hal-00463216}

Submitted on 3 May 2021

HAL is a multi-disciplinary open access archive for the deposit and dissemination of scientific research documents, whether they are published or not. The documents may come from teaching and research institutions in France or abroad, or from public or private research centers.
L'archive ouverte pluridisciplinaire HAL, est destinée au dépôt et à la diffusion de documents scientifiques de niveau recherche, publiés ou non, émanant des établissements d'enseignement et de recherche français ou étrangers, des laboratoires publics ou privés. 


\title{
Polarization-dependent extraction properties of bare fiber probes
}

\author{
T. Grosjean, ${ }^{1, *}$ M. Mivelle, ${ }^{1,2}$ and G. W. Burr ${ }^{2}$ \\ ${ }^{1}$ Département d'Optique P.M. Duffieux, Institut FEMTO-ST, CNRS UMR 6174, Université de Franche-Comté, \\ 16 Route de Gray, 25030 Besançon Cedex, France \\ ${ }^{2}$ IBM Almaden Research Center, D2/K13E, 650 Harry Road, San Jose, California 95120, USA \\ *Corresponding author: thierry.grosjean@univ-fcomte.fr
}

\begin{abstract}
Despite their modest spatial resolution, uncoated tapered fiber probes are now widely used by the nanooptics community for mapping, with scanning near-field optical microscopy (SNOM), the nonradiative fields at the surface of optical and plasmonic microstructures and nanostructures. Given the significant complexity of the vectorial optical phenomena associated with subwavelength structures, the correct interpretation of SNOM acquisitions requires a complete and accurate understanding of the intrinsic image-formation procedure. In this theoretical study, we show that the SNOM imaging process with uncoated tapered fiber probes is highly polarization dependent and that the dominant effect is, surprisingly, the choice of optical fiber from which the tapered probe was fabricated. We demonstrate that although a tapered monomode fiber is unable to collect the component of the vector electric field parallel to the tip axis, a tapered multimode fiber can successfully collect all the three field components. However, we show that the signal from the longitudinal field component is collected only $10 \%$ as efficiently as the signal from the two transverse field components.
\end{abstract}

Scanning near-field optical microscopy (SNOM) is a well-established technique for imaging the finer details of samples. The ability of the SNOM probes to emit and collect the evanescent field in the close neighborhood of nanostructures offers the possibility of achieving subwavelength resolution imaging inaccessible by conventional far-field microscopies. Among the various configurations implemented over the past 25 years, the collection-mode SNOM [1] has grown rapidly as micro-optics has given way to nanooptics. Dielectric bare fiber tips have become a fundamental research tool in such near-field optical experiments. The main reason for this popularity is that these sharp probe tips offer both simple fabrication (with either heating-pulling or chemical etching techniques), as well as low probe-to-sample light interaction [2].

Recent studies have shown that bare fiber probes are mostly sensitive to the optical electric field, with little or no sensitivity to the optical magnetic field [3-5]. Although subwavelength-scale optics immediately implies strong local variations in the vector light orientation, only a few studies have been devoted to the coupling between vector electric fields and uncoated fiber probes. Worse yet, these studies present results that would seem to be directly contradictory. On the one hand, it has been experimentally shown that bare fiber probes are unable to collect the longitudinal electric field parallel to the tip axis [6]. This polarization-filtering property represents the loss of valuable information, since longitudinal optical fields, nonnegligible only at submicron scale, carry unique and nonradiative information about fine sample structure. On the other hand, it has been shown that bare fiber tips can collect the longitudinal electric field in the context of a multi-heterodyne detection technique [7].
In this Letter, we resolve this apparent contradiction by using rigorous numerical methods to quantitatively analyze the polarization-dependent light extraction properties of sharp uncoated fiber probes. We show that the optical fiber itself, through the polarization and cutoff properties of the modes it supports, defines the sensitivity of the probe to the vector electric field.

In this study, we cascade two different numerical approaches to model the optical collection process of uncoated fiber tips. The first tip collection step (light scattering by the apex and channeling through the taper) is numerically investigated using the finitedifference time-domain (FDTD) method [8]. The accurate simulation of the entire $3 \mathrm{D}$ elongated tip has been rendered possible with the use of a BlueGene/L supercomputer [9]. The second step, of the tip-to-fiber optical coupling, is simulated with the well-known overlap integral method [10]. Here we assume only that the detector is sensitive to the entire energy flow through the fiber output cross section and that there are no losses in the fiber other than those associated with mode-matching.

The study of the probe sensitivity with respect to the three impinging electric field components is carried out by numerically simulating the efficiency with which orthogonal single dipoles located at the sharp tip couple light into and through the entire $30 \mu \mathrm{m} \mathrm{ex}-$ tent of the taper. Dipole current sources are highly appropriate test objects for polarization-dependent systems, since they are known to produce localized purely linearly polarized light along their dipole moment [11]. The distributed power collection process between these elementary nanosources and the tapered uncoated fiber probe is fully and rigorously simulated with the FDTD part of our simulations corresponding directly to the experimental detection of 
fluorescence from oriented single molecules.

The model used for the calculation of the tip collection process is shown in Fig. 1(a) consisting of a volume spanning $\pm 5 \mu \mathrm{m}$ in $X$ and $Y$ about the apex of an uncoated fiber tip. The apex, with a tip radius of 25 $\mathrm{nm}$, is located at $X=Y=Z=0$, and the simulation spans 0.5 um below the tip in air and terminates $29.5 \mu \mathrm{m}$ into the body of the tip. The fiber tip geometry considered in this study is that of a tapered fourmode fiber with core and cladding optical indices of 1.4574 and 1.4529 , respectively, and a core diameter of $5 \mu \mathrm{m}$. The taper angle is $20^{\circ}$ consistent with the angles achieved with the widely used tube etching process of fiber tip fabrication [12]. The wavelength is $633 \mathrm{~nm}$ in all calculations. The length of the guiding part of the tip (with nontapered core) is about $15 \mu \mathrm{m}$ long (24 wavelengths) in our simulations. All six boundaries of the computation volume are terminated with convolutional perfectly matched layers [13] in order to avoid parasitic unphysical reflections around the probe. The nonuniform grid resolution varies from $25 \mathrm{~nm}$ for portions at the periphery of the simulation to $5 \mathrm{~nm}$ in the region immediately around the apex ( $\pm 100 \mathrm{~nm}$ in $X, Y$ and -150 to $400 \mathrm{~nm}$ in $Z$ ). The resulting simulations are $432 \times 432 \times 1288$ grid cells in size and require $85 \mathrm{~min}$ of simulation time to converge on a BlueGene/L partition with 1024 dualprocessor nodes.

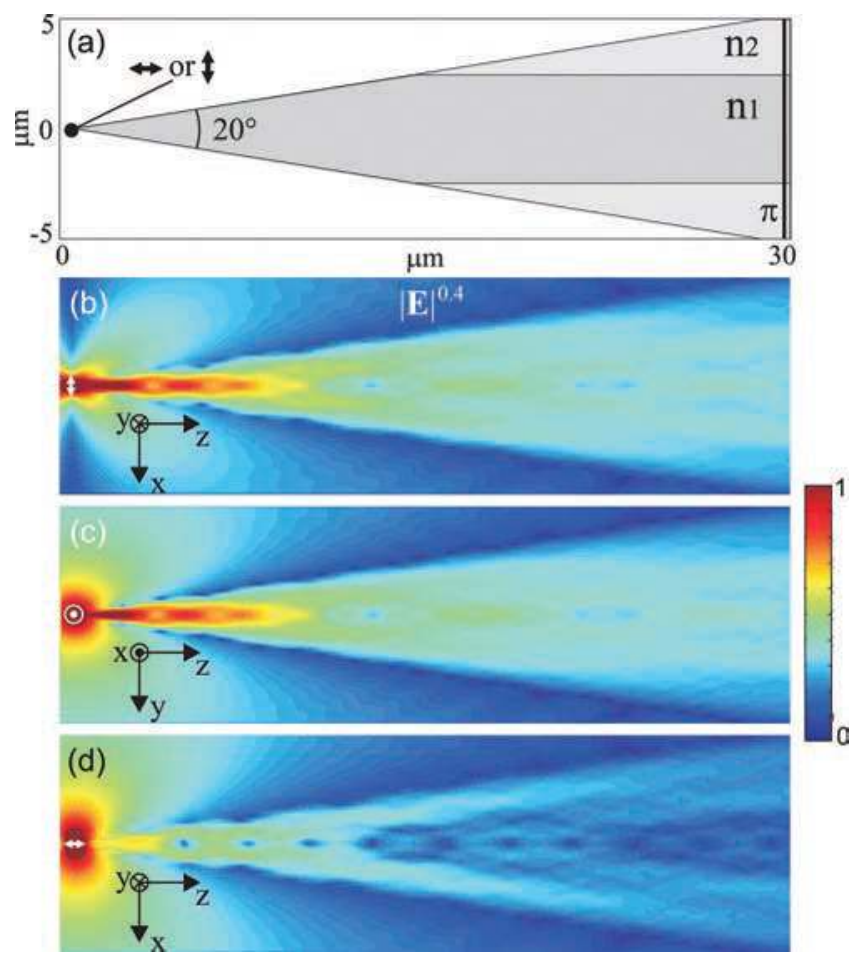

Fig. 1. Light collection into a four-mode tapered optical fiber with a dipolar excitation. (a) Scheme of the theoretical configuration. (b) and (c) Electric field amplitude (to the power 0.4$)$ in the (b) $(X Z)$ and (c) $(Y Z)$ planes with a transverse dipole excitation along $X$. (d) Electric field amplitude (to the power 0.4$)$ in the $(X Z)$ plane with a longitudinal dipole excitation.
To excite the uncoated fiber probe in the extraction mode, a single dipole is placed directly under the tip at a distance of $10 \mathrm{~nm}$ and excited with a simple monochromatic sinusoid. Figures 1(b) and 1(c) show the resulting field amplitude distributions along the $(X Z)$ and $(Y Z)$ planes, respectively, for a dipole oriented in the transverse $X$ direction. Figure 1(d) displays one cross section of the rotationally symmetric distribution of the field amplitude for a dipole oriented along the longitudinal $Z$ direction. In these figures, the color scale is mapped not to field amplitude, but to field amplitude raised to the power 0.4 , in order to provide a better view of the light distributions in the taper.

The simulations reveal two different field distributions within the tip for the two orthogonal dipole directions. For the transverse dipole [Figs. 1(b) and 1(c)], the collected light stays well concentrated along the tip axis while propagating toward the fiber core. In contrast, for the other dipole orientation [Fig. $1(\mathrm{~d})$ ], the field is mostly located close to the conical end of the tip and reaches a minimum along the tip axis. Further studies (not detailed here) show that the transverse dipole leads to a linearly polarized beam within the taper, whereas the longitudinal dipole generates a radially polarized doughnut beam.

The power collected by the tip for the two dipole orientations can be obtained by calculating the Poynting vector flow through the tip output plane [ $\pi$-plane; see Fig. 1(a)]. We find that the collection efficiency of the tip excited with the longitudinal dipole is only $27 \%$ as large as the efficiency achieved with the transverse dipole. Note that this collection efficiency represents both the influence of the tip on the total amount of power radiated by the dipole in its environment, as well as the coupling of the resulting polarized light field into the tip. Thus the taper itself is fully capable of extracting a signal from all the three dipole sources, except that the signal from the longitudinal dipole is noticeably attenuated (by a factor $R_{\text {tip }}=3.70$ ) with respect to the signal from the transverse dipole.

Owing to its geometrical and physical properties, the optical fiber described above supports only four modes. These include the fundamental mode, $\mathrm{HE}_{11}$, which has no cutoff, as well as the three annular modes $\mathrm{TM}_{01}, \mathrm{TE}_{01}$, and $\mathrm{HE}_{21}$ with radial, azimuthal, and hybrid polarizations, respectively. The three latter modes have almost the same cutoff frequencies for the low guiding optical fiber of interest [14].

The energy transfer between the signal that leaves the tip body and these fiber modes can be described in terms of the coupling coefficients $C_{m}$, defined as [10] $C_{m}=P_{m} / P_{i}$, where $P_{m}$ and $P_{i}$ are the powers carried by the mode of type $m$ and by the total collected signal, respectively. Here, we have $m=\mathrm{HE}_{11}, \mathrm{TM}_{01}$, $\mathrm{TE}_{01}$, and $\mathrm{HE}_{21} . P_{m}$ and $P_{i}$ can be obtained from the Poynting vector flow through the fiber cross section:

$$
P_{i}=\frac{1}{2} \mathfrak{R e} \iint r \mathrm{~d} r \mathrm{~d} \theta\left(\vec{E}_{i} \times \vec{H}_{i}^{*}\right) \cdot \vec{e}_{z},
$$




$$
P_{m}=\frac{1}{2} \mathfrak{R e} \iint r \mathrm{~d} r \mathrm{~d} \theta\left(a_{m} \vec{E}_{m} \times b_{m}^{*} \vec{H}_{m}^{*}\right) \cdot \vec{e}_{z} .
$$

Here the unit vector $\vec{e}_{z}$ defines the direction of the fiber axis, and constants $a_{m}$ and $b_{m}$ are the components of the input electric and magnetic field distributions, respectively, of the $m$-mode orthogonal basis. These distributions are based on the overlap integrals between the beam leaving the tip $\left(\vec{E}_{i}, \vec{H}_{i}\right)$ and the mode field distributions $\left(\vec{E}_{m}, \vec{H}_{m}\right)$ :

$$
\begin{aligned}
& a_{m}=\frac{\iint r \mathrm{~d} r \mathrm{~d} \theta\left(\vec{E}_{i} \times \vec{H}_{m}^{*}\right) \cdot \vec{e}_{z}}{\iint r \mathrm{~d} r \mathrm{~d} \theta\left(\vec{E}_{m} \times \vec{H}_{m}^{*}\right) \cdot \vec{e}_{z}}, \\
& b_{m}=\frac{\iint r \mathrm{~d} r \mathrm{~d} \theta\left(\vec{E}_{m}^{*} \times \vec{H}_{i}\right) \cdot \vec{e}_{z}}{\iint r \mathrm{~d} r \mathrm{~d} \theta\left(\vec{E}_{m}^{*} \times \vec{H}_{m}\right) \cdot \vec{e}_{z}} .
\end{aligned}
$$

Table 1 shows the coefficients $C_{m}$ for the two perpendicular dipole orientations. For clarity, here we write zero for any mode-coupling coefficient smaller than $10^{-4}$. We see that only the fundamental mode is excited when the dipole is oriented along $X$ (or $Y$ ) whereas a dipole parallel to $Z$ excites only the annular mode $\mathrm{TM}_{01}$.

Because the calculated coupling coefficients show different values for the two dipole orientations, we can deduce that the fiber itself plays a significant role in the image-formation procedure of the collectionmode SNOM. A tapered monomode fiber collects only the transverse field and filters out the longitudinal optical electric field from the sample, because any energy successfully coupled from the longitudinal fields at the sample into the tip body cannot be subsequently coupled to the fundamental $\mathrm{HE}_{11}$ fiber mode. Only tapered multimode fibers, which allow both $\mathrm{HE}_{11}$ and $\mathrm{TM}_{01}$ to propagate, allow the extraction of the full 3D vector field of a given sample. This observation helps explain why some experiments show no collection of the longitudinal field (with tips constructed from the monomode fiber) while others can reveal the successful collection of this $Z$-oriented field component (using the multimode fiber).

For these latter tips, we see from Table 1 that the imperfect matching between the power reaching the core from the tip and these fiber modes further re-

Table 1. Normalized Mode-Coupling Coefficients $C_{m}$ between the Power Reaching the Core due to a $\mathrm{Di}$ pole at the Tip (Oriented along Either $\hat{x}, \hat{y}$, or $\hat{z}$ ) and the Four Modes Supported by the Few-Mode Fiber

\begin{tabular}{lllcc}
\hline & $\mathrm{HE}_{11}$ & $\mathrm{TM}_{01}$ & $\mathrm{TE}_{01}$ & $\mathrm{HE}_{21}$ \\
\hline$\vec{p} \|(0 x)$ & 0.48 & 0 & 0 & 0 \\
$\vec{p} \|(0 z)$ & 0 & 0.36 & 0 & 0 \\
\hline
\end{tabular}

duces, by a factor $K_{m}=C_{\mathrm{HE}_{11}} / C_{\mathrm{TM}_{01}}=1.33$, the signal from the longitudinal dipole relative to the signal from the transverse dipole. The combination of the tip-to-core attenuation mentioned earlier $\left(R_{\text {tip }}=3.70\right)$ and this mode-matching component $\left(K_{m}\right)$ results in a total attenuation of the signal due to the longitudinal field at the tip (relative to the signal due to an otherwise identical transverse field) by a factor 4.93 .

Note that this value includes the modification of the total amount of power radiated by the dipole sources due to the presence of the tip itself and thus directly represents the expected results for the SNOM imaging of oriented fluorophores. Further FDTD simulations have shown that the total power emitted by the longitudinal dipole when it is near the tip is 2.03 times larger than that radiated by the transverse dipole in the same position. Thus the longitudinal-to-transverse field attenuation factor for freely propagating vectorial fields collected into such a multimode tapered fiber tip may in fact be larger than 10. This factor will be important to characterize empirically for accurate SNOM image interpretation. However, as we have shown here, the use of a multimode fiber is essential in order to have any signal originating from longitudinal fields at the tip reach the far end of the fiber.

This work was funded by the Agence Nationale de la Recherche under contract no. ANR07-NANO-036. It was supported by the "Pôle de compétitivité Microtechnique."

\section{References}

1. E. Betzig, M. Isaacson, and A. Lewis, Appl. Phys. Lett. 51, 2088 (1987).

2. J. Weeber, F. de Fornel, and J. Goudonnet, Opt. Commun. 126, 285 (1996).

3. J.-J. Greffet and R. Carminati, Prog. Surf. Sci. 56, 133 (1997).

4. N. Gregersen, B. Tromborg, and S. I. Bozhevolnyi, Appl. Opt. 45, 8739 (2006).

5. N. Louvion, D. Gerard, J. Mouette, F. de Fornel, C. Seassal, X. Letartre, A. Rahmani, and S. Callard, Phys. Rev. Lett. 94, 113907 (2005).

6. T. Grosjean and D. Courjon, Phys. Rev. E 67, 046611 (2003).

7. P. Tortora, R. Dändliker, W. Nakagawa, and L. Vaccaro, Opt. Commun. 259, 876 (2006).

8. A. Taflove and S. Hagness, Computational Electrodynamics: the Finite-Difference Time-Domain Method (Artech House, 2005).

9. A. Gara, M. Blumrich, D. Chen, G. Chiu, P. Coteus, M. Giampapa, R. Haring, P. Heidelberger, D. Hoenicke, G. Kopcsay, T. Liebsch, M. Ohmacht, B. SteinmacherBurow, T. Takken, and P. Vranas, IBM J. Res. Dev. 49, 195 (2005).

10. Thomson-CSF, L'Optique Guidée Monomode et Ses Applications (Masson, 1983), Vol. 15, pp. 663-666.

11. J. Jackson, Classical Electrodynamics (John Wiley \& Sons, 1999).

12. R. Stöckle, C. Fokas, V. Deckert, R. Zenobi, B. Sick, B. Hecht, and U. Wild, Appl. Phys. Lett. 75, 160 (1999).

13. J. Roden and S. Gedney, Microwave Opt. Technol. Lett. 27, 334 (2000).

14. D. Marcuse, Light Transmission Optics (Van Nostrand Reinhold, 1972), Chap. 8, pp. 302-303. 